Commun. math. Phys. 6, 233-247 (1967)

\title{
The Heisenberg Ferromagnet as a Quantum Field Theory
}

\author{
R. F. StreateR* \\ Institute des Hautes Etudes Scientifiques \\ Bures-sur-Yvette
}

Received May 5, 1967

\begin{abstract}
We consider a lattice of spin $\frac{1}{2}$ ions, described by the discrete form of the current commutation relations $J_{i}^{\alpha} J_{(i)}^{\alpha}=\frac{1}{2},\left[J_{i}^{\alpha}, J_{j}^{\beta}\right]=i \delta_{i j} \varepsilon^{\alpha \beta \gamma} J_{i} \gamma$ where $\alpha=1,2,3$ and $i$ label the lattice sites. The algebra is realized as the Clifford algebra $\mathfrak{A}$ over a Hilbert space. The equations of motion are specified by a formal Hamiltonian of the Heisenberg form: $\mathrm{H}=\sum_{i, j} f_{i j} \underline{J}_{i} \cdot \underline{J}_{j}$, where $f_{i j} \leqq 0$ and only a finite number $Q$ of ions are linked to any given lattice site. We prove that the Hamiltonian is non-negative in a representation of $\mathfrak{l}$, and has a ground state $\Omega$ exhibiting ferromagnetism. The time displacement group acts continuously on $\mathfrak{A}$, inducing automorphisms. $\mathfrak{U}$ is asymptotically abelian with respect to the space translations of the lattice.

The model is an example of an algebraic quantum field theory and possesses a broken symmetry, the rotation group $0(3)$. The consequent Goldstone theorem is proved, namely, there is no energy gap in the spectrum of $H$.
\end{abstract}

\section{Introduction and Summary}

In this paper we apply the ideas of local quantum theory [1-4] to the theory of the Heisenberg ferromagnet [5]. The intention is to discuss the axioms of quantum statistical mechanics $[6-8]$ with reference to this particular model.

Denote by $Z^{d}$ (where $Z$ is the group of integers) the regular arrays of points in $d$ dimensions, $d=1,2,3$. The lattice is invariant under translations by $Z^{d}$. The points of $Z^{d}$ will be called lattice sites. At each lattice site is placed an ion with spin $\frac{1}{2}$. That is, the states of a single ion $i \in Z^{d}$ can be described by the vectors in a two-dimensional Hilbert space $\mathscr{H}_{i}$ carrying the fundamental spinor representation of $\mathrm{SU}_{2}$, the covering group of the rotation group in three dimensions (we use the same description of spin whether the ions are arranged in a chain, a plane or a cube). In the model under consideration the motion of the ions, even the lattice vibrations, are ignored. The "observables" describing an ion $i$ comprise the three Pauli matrices $\sigma_{i}^{1}, \sigma_{i}^{2}, \sigma_{i}^{3}$ where $J_{i}=\frac{1}{2} \sigma_{i}$ measures

* Permanent address: Mathematics Dept., Imperial College, London S. W. 7. 
the spin in a fixed but arbitrary frame of reference, assumed chosen the same for each ion. The observables for the lattice site $i$ generate a $C^{*}$-algebra $\mathfrak{A}_{i}$, equal to $B\left(\mathscr{H}_{i}\right)$, the set of all linear operators on $\mathscr{H}_{i}$.

By taking tensor products of $\mathfrak{A}_{i}$ we can define the $C^{*}$-algebra $\mathfrak{A}(K)$ for any finite subset $K \subset Z^{d}$ (see Section 2). These algebras correspond to the local algebras of quantum field theory. The collection of all the local algebras generate by the inductive limit [9] a unique abstract $C^{*}$-algebra $\mathfrak{A}$, the algebra of all quasi local observables. $\mathfrak{A}$ is isomorphic to the Clifford algebra over a Hilbert space $[10,11,12]$, that is, the $C^{*}$-algebra of the anti-commutation relations [13]. But for the magnet the physical interpretation is entirely different; the localization in real space given by the map $K \rightarrow \mathfrak{A}(K)$ from subsets of $Z^{d}$ to subalgebras is such that operators for different regions commute for the magnet, whereas the localization chosen for a fermi-field is such that the fields anti-commute at space-like separated points. Another difference is that the spins of the lattice sites are observables, but the fermi-fields themselves are not, so that the algebra of observables in that case is a subalgebra of $\mathfrak{A}$. We summarize the properties of $\mathfrak{A}$ proved in Section 2 .

(i) To each finite $K \subset Z^{d}$ there is an assigned subalgebra $\mathfrak{A}(K) \subset \mathfrak{A}$, and $\mathfrak{A}=\bigcup_{K} \mathfrak{A}(K)$ (local structure).

(ii) If $K \supset J, \mathfrak{A}(K) \supset \mathfrak{A}(J)$ (monotony).

(iii) If $K \cap J=\theta$, then $[\mathfrak{A}(K), \mathfrak{A}(J)]=0$ (local commutativity).

(iv) There exists a homomorphism $g \rightarrow \alpha(g)$ of $Z^{d}$ into Aut(2) such that if $A \in \mathfrak{U}(K), A_{g} \equiv \alpha(g) A \in \mathfrak{A}(g K)$ for all $g \in Z^{d}, K \subset Z^{d}$. The automorphisms $\alpha(g)$ of $\mathfrak{A}$ correspond to spatial displacements of the lattice sites.

(v) If $A, B$ in $\mathfrak{A}$, then $\lim _{g \rightarrow \infty}\left\|\left[A, B_{g}\right]\right\|=0$ ( $\mathfrak{U}$ is asymptotically abelian).

These properties are purely kinematical, that is, hold independently of the choice of Hamiltonian. In Section 3 we prove that the Heisenberg form for the Hamiltonian can be made into a non-negative self-adjoint operator $H$ in a certain faithful representation $\pi_{0}$ of $\mathfrak{A}$, chosen by physical intuition. Defining $U(t)=e^{i H t}$ it is proved that if $A \in \mathfrak{A}$.

(vi) $U(t) \pi_{0}(A) U^{-1}(t) \in \pi_{0}(\mathfrak{R})$.

(vii) Defining $A_{t}=\pi_{0}^{-1}\left(U(t) \pi_{0}(A) U^{-1}(t)\right)$ we have

$$
\left\|A-A_{t}\right\| \rightarrow 0 \text { as } t \rightarrow 0 \text {. }
$$

The representation $\pi_{0}$ is given by a cyclic vector $\Omega$, invariant under $U(t)$, with the physical interpretation as the ferromagnetic ground state, with all the spins aligned in the same direction of space. The carrier space $\mathscr{H}$ for $\pi_{0}$ can be written $\mathscr{H}=\oplus \mathscr{H}_{n}$; in $\mathscr{H}_{n}$, the vectors are characterized by having exactly $n$ spins flipped from the ground state, and physically correspond to the presence of $n$ spin-waves. We show $\mathscr{H}_{n}$ is invariant under $U(t)=e^{i H t}$, that is, the number of spin-waves is a 
constant of the motion. Moreover, the maximum energy per spin wave must be less than $\sqrt{10} f Q$.

In Section 4 we prove there is no energy gap in the spectrum of $H$. The technique follows the usual method of proof of the Goldstone theorem [14] making use of an idea to be found in [15]. There is a continuous automorphism of $\mathfrak{A}$ induced by rotating the spins, which is not implemented in the representation $\pi_{0}$. This automorphism commutes with space-time translation and maps each of the local algebras $\mathfrak{A}(J)$ onto itself. Thus rotations have the general properties of a spontaneously broken symmetry [16]. Finally we remark that macroscopic properties of the magnet such as direction of magnetization and orientation of the boundaries of magnetic domains, can be described by parameters labelling inequivalent representations of $\mathfrak{A}$. At infinite temperature the thermodynamic state is the central trace on $\mathfrak{A}$, and defines the hyperfinite $I I_{1}$ factor.

\section{The $C^{*}$-algebra of the Spin $\frac{1}{2}$ Lattice}

If $K=\left(i_{1}, i_{2}, \ldots, i_{k}\right) \subset Z^{d}$, define a $2^{k}$-dimensional Hilbert space

$$
\mathscr{H}_{K}=\mathscr{H}_{i_{1}} \bar{\otimes} \mathscr{H}_{i_{2}} \bar{\otimes} \cdots \bar{\otimes} \mathscr{H}_{i_{k}} \text {. }
$$

Clearly, $\mathscr{H}_{K}$ carries the tensor product of the spin $\frac{1}{2}$ representation of $S U_{2}$ with itself $k$ times. A complete orthonormal basis in $\mathscr{H}_{i}$ consists of two vectors, which may be taken as the eigenstates $| \pm\rangle_{i}$ of $\sigma_{i}^{3}=\left({ }^{1}-1\right)$. An orthonormal basis in $\mathscr{H}_{K}$ is then given by the $2^{k}$ distinct products

$$
\left| \pm i_{1}, \pm i_{2}, \ldots \pm i_{k}\right\rangle=| \pm\rangle_{i_{1}} \otimes| \pm\rangle_{i_{2}} \otimes \cdots \otimes| \pm\rangle_{i_{k}}
$$

In $\mathscr{H}_{K}$ we define the operators

$$
J_{i}=1 \otimes 1 \cdots \otimes 1 \otimes \frac{1}{2} \sigma_{i} \otimes 1 \cdots \otimes 1 .
$$

These operators satisfy the current commutation relations

$$
\left[J_{i}^{\alpha}, J_{j}^{\beta}\right]=i \varepsilon^{\alpha \beta \gamma} \delta_{\imath j} J_{\imath}^{\gamma} .
$$

The vector generator of the representation of $S U_{2}$ carried by $\mathscr{H}_{K}$ is then ${ }^{1}$

$$
\underline{J}_{K}=\sum_{i \in K} \underline{J}_{i} \text {. }
$$

The $C^{*}$-algebra generated by all $\underline{J}_{i}=\left(J_{i}{ }^{1}, J_{i}{ }^{2}, J_{i}{ }^{3}\right)$ for $i \in K$ will be called the algebra of observables $\mathfrak{A}(K)$ associated with the points of $K$. Since all the spaces $\mathscr{H}_{K}$ are finite-dimensional, we have $\mathfrak{A}(K)=B\left(\mathscr{H}_{K}\right)$. If $I \subset K$ any operator in $\mathfrak{A}(I)$ may be identified with an operator in $\mathfrak{A}(K)$, by writing $\mathscr{H}_{K}=\mathscr{H}_{I} \bar{\otimes} \mathscr{H}_{K-I}$, and identifying $A \in \mathfrak{A}(I)$ with $A \otimes \mathbf{1}_{K-I}$, where for any $J \subset Z^{d}, \mathbf{1}_{J}$ is the identity operator in $\mathscr{H}_{J}$. This

\footnotetext{
$1 \quad J$ denotes the 3 -vector $\left(J^{1}, J^{2}, J^{3}\right)$.
} 
induces a natural norm preserving injection of the algebras $i_{I K}: \mathfrak{A}(I) \rightarrow$ $\rightarrow \mathfrak{A}(K)$.

We define the abstract $C^{*}$-algebra of the spin $\frac{1}{2}$ lattice to be the inductive limit [9] of all the algebras $\mathfrak{A}(J), J \subset Z^{d}$ and $J$ finite, relative to the injection $i_{J K}$, and we write $\mathfrak{A}\left(Z^{d}\right)$ for this limit. Clearly, one could analogously define an algebra whatever the spin of the ions on the lattice. The algebra $\mathfrak{A}\left(Z^{d}\right)$ can be regarded as the norm closure of the union of subalgebras $\mathfrak{A}(J)$ for all finite subsets $J$ of $Z^{d}$. This proves axiom (i). Axioms (ii) and (iii) also obviously hold. The transformation $J_{i}^{\alpha} \rightarrow J_{g i}^{\alpha}$ for all $\alpha=1,2,3$, for all $i \in Z^{d}$ and for some $g \in Z^{d}$ induces an automorphism of $\mathfrak{A}$, in accordance with axiom (iv). To prove property (v), choose $A$ and $B$ and $\varepsilon>0$. Since $\mathscr{B}=\bigcup_{J} \mathfrak{A}(J)$ is dense in $\mathfrak{A}$ there exist operators $A_{1} \in \mathscr{B}, C \in \mathscr{B}$ such that $\left\|A-A_{1}\right\|<\frac{\varepsilon}{4\|B\|}$ and $\|B-C\|<\frac{\varepsilon}{4\left\|A_{1}\right\|}$. If $|g|$ is sufficiently large, $g \in Z^{d}$, then $\left[A_{1}, C_{g}\right]=0$, by axiom (iii). But then

This proves $(\mathrm{v})$.

$$
\begin{gathered}
\left\|\left[A, B_{g}\right]\right\| \leqq\left\|\left[A-A_{1}, B_{g}\right]\right\|+\left\|\left[A_{1},(B-C)_{g}\right]\right\|+\left\|\left[A_{1}, C_{g}\right]\right\| \leqq \\
\leqq 2 \frac{\varepsilon}{4} \frac{\|B\|}{\|B\|}+2 \frac{\varepsilon}{4} \frac{\left\|A_{1}\right\|}{\left\|A_{1}\right\|}=\varepsilon .
\end{gathered}
$$

A large set of irreducible representations of $\mathfrak{A}$ but not all ([12], Prop. 2.12) can be obtained by considering the infinite tensor product [17] $\tilde{\mathscr{H}}=\prod_{i \in Z^{a}} \otimes \mathscr{H}_{i}$. The elements of $\tilde{\mathscr{H}}$ are represented by sequences $\psi=\left\{\psi_{i}\right\}$ with $\psi_{i} \in \mathscr{H}_{i}$, such that $\prod_{i \in Z^{a}}\left\|\psi_{i}\right\|^{2}$ is convergent to $\|\psi\|^{2}$ say, in the sense that to any $\varepsilon>0$ there exists a finite subset $K \subset Z^{d}$ such that $\left|\left(\prod_{i \in K_{1}}\left\|\psi_{i}\right\|^{2}\right)-\|\psi\|^{2}\right|<\varepsilon$ for all finite subsets $K_{1} \subset Z^{d}$ containing $K$. The scalar product of two vectors $\psi=\left\{\psi_{i}\right\}$ and $\varphi=\left\{\varphi_{i}\right\}$ in $\tilde{\mathscr{H}}$ is defined to be

$$
\left.\begin{array}{rl}
(\psi, \varphi) & =\prod_{i \in Z^{d}}\left(\psi_{i}, \varphi_{i}\right) \text { if this converges in the above sense } \\
& =0 \text { otherwise. }
\end{array}\right\}
$$

We need consider only those sequences $\left\{\psi_{i}\right\}$ with no zero elements, since if $\psi_{i}=0$ for some $i$ then $\psi=\left\{\psi_{i}\right\}$ represents the zero vector in $\tilde{\mathscr{H}}$, and the same goes for sequences $\left\{\psi_{i}\right\}$ such that $\Pi\left\|\psi_{i}\right\|^{2}=0$. Modulo the sequences of zero norm, the sequences define a non-separable Hilbert space $\tilde{\mathscr{H}}$.

If $\left\{\varphi_{i}\right\},\left\{\psi_{i}\right\}$ represent distinct vectors in $\tilde{\mathscr{H}}$, von NEumann writes $\varphi \sim \psi$ if $\Pi\left(\varphi_{i}, \psi_{i}\right)$ converges, and shows that this is an equivalence relation. The set $b(\psi)$ of sequences equivalent to $\psi$ form a separable subspace of $\Pi_{\otimes} \mathscr{H}_{i}$, known as the incomplete tensor product and written $\mathscr{H}^{b}=\Pi_{\circledast}^{b} \mathscr{H}_{i}$. 
It is trivial to show that for any finite set $K \subset Z^{d}$ we may write

$$
\mathscr{H}^{b}=\mathscr{H}_{K} \bar{\otimes} \prod_{i \in Z^{d}-K}^{b^{\prime}} \otimes \mathscr{H}_{i}
$$

where $\mathscr{H}_{K}$ is given by (1), and $b^{\prime}$ is the equivalence class of the sequence $\left\{\psi_{i}\right\}_{i \in Z^{\alpha}-K}$ obtained from $\psi$ by omitting elements in $K$. One may then define a natural norm-preserving map from $\mathfrak{A}(K)$ into $B\left(\mathscr{H}^{b}\right)$ by

$$
A \rightarrow A \otimes 1_{b^{\prime}}=\bar{A}
$$

where $\mathbf{1}_{b^{\prime}}$ is the unit in $\mathscr{H}^{b^{\prime}}$. The $C^{*}$-algebra generated by all such $\bar{A}$ defines an irreducible representation $\pi^{b}$ of $\mathfrak{Q}\left(Z^{d}\right)$, since the $W^{*}$-algebra generated by it is $B\left(\mathscr{H}^{b}\right)$ [17].

If $\prod_{i \in Z^{d}}\left|\left(\varphi_{i}, \psi_{i}\right)\right|$ converges we say the sequences $\left\{\varphi_{i}\right\}$ and $\left\{\psi_{i}\right\}$ are weakly equivalent [17], written $\varphi \stackrel{\mathfrak{w}}{\sim} \psi$. Then every sequence in $b(\varphi)$ is weakly equivalent to every sequence in $b(\psi)$ and we write $b(\psi) \stackrel{w}{\sim} b(\varphi)$. The following theorem holds ([12], see also [18, 19]).

Theorem 1. Two representations $\pi^{b_{1}}$ and $\pi^{b_{2}}$ of $\mathcal{A}\left(Z^{d}\right)$ are equivalent if and only if $b_{1} \stackrel{w}{\sim} b_{2}$.

The central problem of the theory is to define a 1-parameter automorphism group of $\mathfrak{A}(Z)$ which, in some representation, has a nonnegative self-adjoint generator. This operator is to be interpreted as the Hamiltonian, and the group as the time-displacement group. In the Heisenberg model the Hamiltonian is defined in terms of the abstract elements of $\mathfrak{A}\left(Z^{d}\right)$, the $\underline{J}_{i}$, by the usual formula

$$
H_{F}=\sum_{\text {all } i, j} f_{i j} \underline{J}_{i} \cdot \underline{J}_{j}
$$

where the $f_{i j}$ are some numbers. We assume $\left|f_{i j}\right| \leqq f$ for all $i, j$. In interesting cases, for example if one has translation invariance, an infinite number of terms enter, and the expression (8) as it stands has no meaning (it will not converge in norm). If all the $f_{i j}$ are negative, then on physical grounds we would expect the energy to be least in states with all the spins aligned; thus the system would exhibit ferromagnetism. Define $\Omega \in \tilde{\mathscr{H}}$ by the sequence $\left\{\psi_{i}\right\}$ where $\psi_{i}=|+\rangle_{i} \in \mathscr{H}_{i}$, so that all the spins are in the direction 3 at all lattice sites. We denote by $\mathscr{H}=\mathscr{H}^{b(\Omega)}$ the corresponding incomplete tensor product, and by $\pi_{0}$ the corresponding representation, which we call the concrete $C^{*}$-algebra of the ferromagnet.

The abstract algebra $\mathfrak{A}$ possesses the following automorphisms: for any $h \in S U_{2}$ let $U_{i}(h)$ be the spin $\frac{1}{2}$ representation on $\mathscr{H}_{i}$. This induces a rotation of any operator $A_{i} \in B\left(\mathscr{H}_{i}\right)$, and tensor products of it $k$ times gives an automorphism of $\mathfrak{A}(K)$. Since this is norm preserving it induces an automorphism $h$ on the inductive limit $\mathfrak{A}$. The automorphism is continuous in the parameters of $S U_{2}$, that is $\left\|A_{h}-A\right\| \rightarrow 0$ as $h \rightarrow e$ $=$ identity in $S U_{2}$. The automorphism $h$ has the property of mapping each local algebra into itself: $\mathfrak{A}(K)_{h}=\mathfrak{A}(K)$, and so restriction of $h$ to 
$\mathfrak{A}(K)$ is implementable in any representation of $\mathfrak{A}$ (since $\mathfrak{A}(K)$ is a finite matrix algebra). Indeed, the generators of this "local" rotation group are given by

$$
\underline{J}^{K}=\sum_{i \in K} \underline{J}_{i} \in \mathfrak{A}(K) \text {. }
$$

Theorem 2. If $h \neq e \in S U_{2}, h$ is not implemented in $\pi_{0}$.

Proof. Let $\pi_{h}(A)=\pi_{0}\left(A_{h}\right)$ be the representation of $\mathfrak{A}$ obtained by using the state $\Omega_{h}=\Pi_{\otimes}\left(U_{i}(h)|+\rangle_{i}\right)$ in the Gelfand-Segal construction. Since clearly $\Omega$ and $\Omega_{h}$ are not $w$-equivalent, $\pi_{h}$ and $\pi_{0}$ are inequivalent, by theorem 1. But if $A \rightarrow A_{h}$ were implementable in $\pi_{0}$, then $\pi_{h}$ would be equivalent to $\pi_{0}$. Therefore $h$ is not implemented in $\pi_{0}$, and similarly, not in any $\pi_{h_{1}}, h_{1} \in S U_{2}$.

The algebra $\mathfrak{A}$ possesses another group $\Gamma$ of automorphisms namely the permutations of $Z^{\mathrm{d}}$. This commutes with any rotation $h$ and is implemented by $W(\gamma)$ say in all the representations $\pi_{h}$ since $\Omega$ and $\Omega_{h}$ are invariant under $\Gamma . \Omega$ is the only vector up to a phase in $\mathscr{H}^{(\Omega)}$ that is invariant under $Z^{\mathrm{d}} \subset \Gamma$ (Proof : obviously the following cluster property holds :

$$
\begin{aligned}
\left(\Omega, J_{1} J_{2} \ldots J_{j} J_{g(j+1)}\right. & \left.\ldots J_{g k} \Omega\right) \rightarrow \\
& \rightarrow\left(\Omega, J_{1} \ldots J_{j} \Omega\right)\left(\Omega, J_{\jmath+1} \ldots J_{k} \Omega\right) \quad \text { as } \quad g \rightarrow \infty,
\end{aligned}
$$

where we have written $J_{i}$ for $\pi^{0}\left(J_{i}\right)$. The proof of uniqueness of $\Omega$ then follows [20], p. 124). This immediately gives an alternative proof of theorem 2. For if $h$ is implemented by a unitary operator $V(h)$ say, then $W(\gamma) V(h) \Omega=V(h) W(\gamma) \Omega=V(h) \Omega$. Thus $V(h) \Omega$ is invariant under $\Gamma$, so

$$
V(h) \Omega=e^{i \alpha} \Omega
$$

by the uniqueness of $\Omega$. But then for $A \in \mathfrak{A}$

$$
\left(\Omega_{\mathrm{h}}, A \Omega_{\mathrm{h}}\right)=(\Omega, A \Omega), \quad h \in S U_{2}
$$

which is clearly false unless $h=e$, proving theorem 2 . We shall see later that the automorphisms $h \in S U_{2}$ of $\mathfrak{A}$ commute with the time-displacements obtained from the Hamiltonian (8), and so define a symmetry of the theory. Since $h$ is not implemented in $\pi_{0}$, it defines a spontaneously broken symmetry [16]. The non-implementability can be seen heuristically by noting that the total spin of the ground state $\Omega$ is infinite, so that (9) diverges as $K \rightarrow \infty$. According to the general theory, the representations $\pi_{h}, h \in S U_{2}$ all are such that the Hamiltonian is non-negative and this is easily checked in this model.

\section{The Hamiltonian}

It is convenient to introduce the operators $J_{1}^{ \pm}=J_{i}^{1} \pm i J_{i}^{2}$; then we have

$$
\left[J_{i}^{+}, J_{j}^{-}\right]=2 \delta_{i j} ;\left[J_{i}^{+}, J_{j}^{3}\right]=-\delta_{i j} J_{i}^{+} ;\left[J_{i}^{-}, J_{j}^{3}\right]=\delta_{i j} J_{i}^{-} .
$$


The state $\Omega$ is then uniquely determined by $J_{i}^{+} \Omega=0, J_{i}^{3} \Omega=\frac{1}{2} \Omega$, an the relations $(10)$.

As shown in [17], a complete orthonormal basis for $\mathscr{H}^{(\Omega)}=\mathscr{H}$ is given by the set of vectors $\psi_{K}$, where $\left(\psi_{K}\right)_{i}=|+\rangle_{i}$ if $i \notin K$ and $\left(\psi_{K}\right)_{i}=|-\rangle_{i}$ if $i \in K$.

The properties (10) then show that

$$
\psi_{K}=J_{i_{1}}^{-} J_{i_{2}}^{-} \ldots J_{i_{k}}^{-} \Omega
$$

where $K=\left(i_{1}, i_{2}, \ldots i_{k}\right)$. We define a dense domain $D_{0}$ in $\mathscr{H}$

$$
D_{0}=\bigcup_{K} \mathfrak{I}(K) \Omega \text {. }
$$

The formal Hamiltonian (8) becomes

$$
H_{F^{\prime}}=\sum_{i, j} f_{i j}\left\{\frac{1}{2}\left(J_{i}^{+} J_{j}^{-}+J_{i}^{-} J_{j}^{+}\right)+J_{i}^{3} J_{j}^{3}\right\} .
$$

Suppose that $f_{i j}=0$ for fixed $i$ except for at most $Q$ values of $j$. Without changing the dynamics we may assume that $f_{i j}=f_{j i}$ and $f_{i i}=0$. The Hamiltonian (13) is only formal since, as it stands, it diverges when acting on $D_{0}$. We now show that a consistent definition of an operator $H$ on $D_{0}$ can be written

$$
H A \Omega=\left[H_{F}, A\right] \Omega
$$

for all $A \in \bigcup_{K} \mathfrak{A}(K)$. The idea is that we "renormalize" $H_{F}$ by subtracting a constant, so that $[H, A]=\left[H_{F}, A\right]$ and $H \Omega=0$. In order for (14) to define an operator, we have to show that if $A \Omega=0$, then $\left[H_{F}, A\right] \Omega=0$. To this end, define an approximate Hamiltonian $H_{F^{\prime}}^{K}$ to be the sum $\sum f_{i j} \underline{J}_{i} \cdot \underline{J}_{j}$, where the sum is taken over the neighbours of the points in $K$. This is then a finite sum, and so is a bounded operator on $\mathscr{H}$. Since $J_{i}^{+} \Omega=0$ for all $i$ and $\Omega$ is an eigen-vector of $J_{i}^{3}$, we see from (13) that $\Omega$ is an eigen-state of $H_{F}^{K}$. Since $H_{F}^{K}$ is bounded, we may modify $H_{F}^{K}$ to $H^{K}$ by adding a finite constant (depending on $K$ ) so that $H^{K} \Omega=0$. We then see that

$$
H^{K} A \Omega=\left[H^{K}, A\right] \Omega .
$$

Now for any $A \in \mathfrak{A}(K)$ it is clear that

$$
\left[H^{K_{1}}, A\right]=\left[H^{K}, A\right]=\left[H_{F}, A\right]
$$

for all $K_{1} \subset K$ since any extra terms in $H^{K_{1}}$ have no neighbours in $K$, and so commute with $A$. If now $A \Omega=0$, then

$$
\left[H_{F}, A\right] \Omega=\left[H^{K}, A\right] \Omega=H^{K} A \Omega-A H^{K} \Omega=0 .
$$

We may therefore unambiguously define an operator $H$ on $D_{0}$ by (14); it is symmetric but not necessarily bounded.

Suppose from now on that all the $f_{i j}$ are negative. Let $\mathscr{H}_{l_{0}}$ be the subspace of $\mathscr{H}$ which is spanned by all the vectors $\psi_{K}$ for some $K$ with $k$ 
elements $\left(i_{1}, \ldots, i_{k}\right)$. The action of the Hamiltonian is $H \psi=\left[H_{F}, J_{i_{1}}^{-} \ldots J_{i_{k}}^{-}\right] \Omega=\sum_{l=1}^{k} J_{i_{1}}^{-} \ldots J_{i_{l-1}}^{-}\left[H_{F}, J_{i_{l}}^{-}\right] J_{i_{l+1}}^{-} \ldots J_{i_{k}}^{-} \Omega$

One easily computes

$$
\left[H, J_{l}^{-}\right]=\sum_{i, j} f_{i j}\left\{\delta_{i \imath} J_{i}^{3} J_{j}^{-}-\delta_{j l} J_{i}^{3} J_{j}^{-}-\delta_{i l} J_{i}^{-} J_{j}^{3}+\delta_{j l} J_{i}^{-} J_{j}^{3}\right\} .
$$

Now $J_{i_{l+1}}^{-} \ldots J_{i_{k}}^{-} \Omega$ is an eigen-vector of all $J_{j}^{3}$ with eigen-value $\pm \frac{1}{2}$. In fact, since all the $i_{1}, \ldots i_{k}$ are different in (17), all the $J_{i}^{3}$ occurring in (18) have eigenvalue $+\frac{1}{2}$ on $J_{i_{l+1}}^{-} \ldots J_{i_{k}}^{-} \Omega$. We see that $H \psi$ is a linear sum of vectors with exactly $k$ products of different "creation operators" $J$ - (if any $J^{-}$is repeated in (11) it gives zero, since $\left(J_{i}^{-}\right)^{2}=0$ ). Therefore the set $\mathscr{H}_{k} \cap D_{0}$ is invariant under $H$. We call $\mathscr{H}_{k}$ the space of states of $k$ spin waves, and write $\mathscr{H}=\bigoplus_{k=0}^{\infty} \mathscr{H}_{k}$.

Theorem 3. The operator $H$ defined by (14) is non-negative.

Proof. Since $H$ is reduced by $\oplus \mathscr{H}_{k}$, it is sufficient to verify that $(\psi, H \psi) \geqq 0$ for each $\psi \in D_{0} \cap \mathscr{H}_{k}$ and then each $k$. Each such $\psi$ has the form (finite sum)

and using (18)

$$
\psi=\sum C_{i_{1} \ldots i_{k}}\left|i_{1} \ldots i_{k}\right\rangle
$$

$$
\begin{aligned}
(\psi, H \psi)= & \sum_{i, j} f_{i j} \sum_{l} \frac{1}{2} C_{i_{1} \ldots i_{k}}\left\{\left\langle\psi \mid i_{1} \ldots \hat{\imath}_{l}, j, \ldots i_{k}\right\rangle \delta_{i i_{l}}+\right. \\
& +\left\langle\psi \mid i_{1}, \ldots \hat{\imath}_{l}, i, \ldots i_{k}\right\rangle \delta_{j i_{l}}-\left\langle\psi \mid i_{1} \ldots \hat{\imath}_{l}, j, \ldots i_{k}\right\rangle \delta_{j i_{l}}- \\
& \left.-\left\langle\psi \mid i_{1} \ldots \hat{\imath}_{l}, i_{1} \ldots i_{k}\right\rangle \delta_{i i_{l}}\right\} .
\end{aligned}
$$

This is exactly the same functional of $C_{i_{1} \ldots i_{k}}$ as is obtained from the manifestly positive operator

$\frac{1}{2} \sum f_{i j}\left(J_{i}^{+} J_{j}^{-}+J_{i}^{-} J_{j}^{+}-J_{i}^{-} J_{i}^{+}-J_{j}^{-} J_{j}^{+}\right)=-\frac{1}{2} \sum f_{i j}\left(J_{i}-J_{j}\right)^{-}\left(J_{i}-J_{j}\right)^{+}$

if one uses the fact that all $i_{1}, \ldots i_{k}$ are distinct. This proves the theorem.

By a theorem of Friedrichs, $H$ possesses a natural self-adjoint extension, which is also non-negative. We denote this extended operator again by $H$. Thus, $U(t)=\exp i H t$ is a 1-parameter group on $\mathscr{H}$, which we take as the time-development of the system. It is not immediately obvious that if $A \in \pi_{0}(\mathfrak{R})$, then $U(t) A U^{-1}(t) \in \pi_{0}(\mathfrak{R})$. We prove this in theorem 5 .

Theorem 4. Restricted to $\mathscr{H}_{k}, H$ is bounded.

Proof. A dense set in $\mathscr{H}_{k}$ is obtained by linear sums $\psi=\sum \alpha_{1} \psi_{1}$, where $I$ has $k$ elements, and all $\left|\alpha_{I}\right|$ are different. We may order the sets $I$ by writing $J>I$ if $\left|\alpha_{\mathrm{I}}\right|>\left|\alpha_{J}\right|$. Then

$$
(\psi, H \psi) \leqq \sum_{I}\left|\alpha_{\mathrm{I}}\right|^{2}\left(\psi_{\mathrm{I}}, H \psi_{\mathrm{I}}\right)+2 \sum_{J>I}\left|\bar{\alpha}_{\mathrm{I}} \alpha_{J}\right|\left|\left(\psi_{\mathrm{I}}, H \psi_{J}\right)\right| .
$$


Looking at (13) we see that $\left(\psi_{\mathrm{I}}, H \psi_{\mathrm{I}}\right)$ is a sum of terms, the number depending on $Q, k$ but not $I$, and each term is bounded by $f$. Hence

$$
\begin{aligned}
\sum_{I}\left|\alpha_{\mathrm{I}}\right|^{2}\left(\psi_{\mathrm{I}}, H \psi_{\mathrm{I}}\right) & \leqq f N(k, Q) \sum_{I}\left|\alpha_{\mathrm{I}}\right|^{2} \\
& =f N(k, Q)\|\psi\|^{2} .
\end{aligned}
$$

In the term $\left(\psi_{I}, H \psi_{J}\right)$, for fixed $I$ only those $J$ give a non-zero contribution that differ from $I$ in one or two places. Hence the number of $J$ 's that enter in $\sum_{J>I}\left|\bar{\alpha}_{I} \alpha_{J}\right|\left|\left(\psi_{I}, H \psi_{J}\right)\right|$ is limited by $k+\frac{k(k-1)}{2}$. For each fixed $J$ the number of terms $i, j$ in the sum $\sum f_{i j}\left(J_{i}-J_{j}\right)^{-}\left(J_{i}-J_{j}\right)^{+} \psi_{J}$ is limited to those which are neighbours of points in $J$ or points in $I$. We may limit this number by a function $M(k, Q)$ independent of $I$ and $J$. Each term is then limited by $f$. Hence

Hence $\|H\| \leqq f N+2 f M$ on $\mathscr{H}_{k}$.

$$
\begin{gathered}
2 \sum_{I<J}\left|\bar{\alpha}_{I} \alpha_{J}\right|\left|\left(\psi_{I}, H \psi_{J}\right)\right| \leqq 2 M(k, Q) f \sum_{I>J}\left|\bar{\alpha}_{I} \alpha_{J}\right|< \\
<2 M(k, Q) f \sum_{I}\left|\alpha_{I}\right|^{2}=2 M f\|\psi\|^{2} .
\end{gathered}
$$

Remark. A more detailed investigation shows that

$$
\begin{gathered}
\|H\| \leqq \sqrt{10} f k Q \text { on } \mathscr{H}_{k} \text { if } 1<k<Q+1 \\
\|H\| \leqq 3 f k Q \text { if } k \geqq Q+1, \text { and }\|H\| \leqq 2 f Q \text { on } \mathscr{H}_{\mathbf{1}} .
\end{gathered}
$$

Corollary. $D_{o}$ is a domain of analytic vectors for $H$, on which $H$ is essentially self-adjoint. The radius of convergence of

$$
\sum \frac{t^{n}}{n !} H^{n} \psi \quad \psi \in D_{0}
$$

is infinite. Each of the subspace $\mathscr{H}_{k}$ is invariant under $U(t)$ (see [21]).

Remark. It is clear that each $\mathscr{H}_{k}$ is invariant under $W(\gamma)$ for all $\gamma \in \Gamma$.

We now come to the key lemma in our discussion of time translation.

Lemma 1. If $|t|<(2 f Q)^{-1}$ we have

$$
U(t) \pi_{0}\left(J_{i}\right) U^{-1}(t) \in \pi_{0}(\mathfrak{Q}) .
$$

Proof. We see for the commutator

$$
\begin{aligned}
{\left[H, J_{l}^{\alpha}\right] } & =\sum f_{i j}\left[J_{i}^{\beta} J_{j}^{\beta}, J^{\alpha}\right] \\
& =\sum f_{i j} \varepsilon_{\alpha \beta \gamma}\left(\delta_{i l} J_{i}^{\beta} J_{j}^{\gamma}+\delta_{i l} J_{i}{ }^{\gamma} J_{j}{ }^{\beta}\right)
\end{aligned}
$$

which is a sum of at most $4 Q$ terms, each quadratic in $J$ (for fixed $\alpha$ each $(\beta, \gamma)$ can have two values, and for fixed $l, f_{i j} \delta_{j l}$ is non-zero for at most $Q$ values of $j$ ). More generally,

$$
\left[H, J_{1}^{\alpha_{1}} \ldots J_{n}^{\alpha_{n}}\right]=\sum_{j=1}^{n} J_{1}^{\alpha_{1}} \ldots\left[H, J_{j}^{\alpha_{j}}\right] J_{j+1}^{\alpha_{j+1}} \ldots J_{n}^{\alpha_{n}}
$$


is a sum of $4 n Q$ terms, each a product of $n+1 J$ 's. Thus, proceeding successively, $[H[H, J]]$ is a sum of $4 Q \cdot 2(4 Q)$ terms, all cubic, and $[H[H[H, J]]]$ is a sum of $4 Q \cdot 2(4 Q) \cdot 3(4 Q)$ terms, all quartic, etc. By induction the $n$ 'th multiple commutator is a sum of $n !(4 Q)^{n}$ terms each of degree $n+1$ in $J$, and involving a product of $n f$ 's. Hence its norm is less than $n !\left(\frac{1}{2}\right)^{n+1} f^{n}(4 Q)^{n}$, since each $J$ has norm $\frac{1}{2}$. Now consider the series

$$
J_{i}^{\alpha}+i t\left[H, J_{i}^{\alpha}\right]+\frac{(i t)^{2}}{2 !}\left[H,\left[H, J_{i}^{\alpha}\right]\right]+\cdots .
$$

Each term in the series is a bounded operator, and the general term is bounded by $\frac{(2 f Q|t|)^{n}}{n !} n !$. Thus the series converges in norm if $|t|<(2 f Q)^{-1}$. But on the dense set $D_{0}$, which is invariant under $J_{i}^{\alpha}$, we have (writing $J_{i}$ for $\pi_{0}\left(J_{i}\right)$ )

$$
U(t) J_{i}^{\alpha} U^{-1}(t) \psi=\left(J_{i}^{\alpha}+i t\left[H, J_{i}^{\alpha}\right]+\cdots\right) \psi
$$

showing that the series (19) converges to $U(t) J_{i} U^{-1}(t)$, which therefore lies in $\pi_{0}(\mathfrak{Q})$. This proves the lemma.

Theorem 5. The time-displacements $A \rightarrow A_{t}=U(t) A U^{-1}(t)$ form a group of automorphisms of $\pi_{0}(\mathfrak{A})$, and therefore of $\mathfrak{A}$, satisfying the continuity axiom (7).

Proof. Any $A \in \bigcup_{K} \mathfrak{A}(K)$ is a finite linear combination of products of operators $J_{i}^{\alpha}$; then applying the lemma, $U(t) A U^{-1}(t) \in \pi_{0}(\mathfrak{R})$, at least for small $t$. Since this is a norm preserving map, it can be extended to an automorphism of $\mathfrak{A}$. The group property $U\left(t_{1}+t_{2}\right)=U\left(t_{1}\right) U\left(t_{2}\right)$ shows that the map $A \rightarrow A_{t}$ is an automorphism for all $t$.

We note that

a) $\mathfrak{A}$ is countably generated,

b) $H$ is self-adjoint in one representation of $\mathfrak{A}$.

These properties imply axiom (vii) [6], which can also be seen directly for this model.

\section{The Goldstone Theorem}

The importance of this theorem is that it enables one to deduce properties of the spectrum of $H$, an unbounded operator not in $\pi_{0}(\mathfrak{A})$, from an apparently unrelated phenomenon, the fact that $\Omega$ is not invariant under rotations. Proofs in other situations can be found in [15, $16,22]$. We must first prove that rotations are a symmetry, that is

Theorem 6. The automorphism $A \rightarrow h A, h \in S U_{2}$, commutes with time translations. 
Proof. It is sufficient to prove this for small $t$. We then see for local $A$, and small $t$

$$
\begin{aligned}
& (h A)(t)=\lim _{n \rightarrow \infty}\left\{h A+i t[H, h A]+\cdots+\frac{i^{n} t^{n}}{n !}[H \ldots[H, h A] \ldots]\right\} \\
& =\lim _{n \rightarrow \infty}\left\{h A+i t\left[H_{F^{\prime}}^{K}, h A\right]+\cdots+\frac{i^{n} t^{n}}{n !}\left[H_{W^{\prime}}^{K}, \ldots\left[H_{W^{\prime}}^{K}, h A\right] \ldots\right]\right\}
\end{aligned}
$$

where $K$, depending on $n$, is chosen large enough. But $H_{F}^{K}$ is rotation invariant, so

$(h A)(t)=\lim _{n \rightarrow \infty}\left\{h\left(A+i t\left[H_{F}^{k}, A\right]+\cdots+\frac{i^{n} t^{n}}{n !}\left[H_{F}^{k}, \ldots\left[H_{F}^{k}, A\right] \ldots\right]\right)\right\}$.

But any automorphism is continuous, so we can invert the order of the limit and the automorphism giving.

$$
(h A)(t)=h A(t) \text {. }
$$

Thus $h t h^{-1} t^{-1}$ is a continuous map $\mathfrak{A} \rightarrow \mathfrak{A}$ coinciding with 1 for local $A$. Hence it is the identity. This proves the theorem. It is a common physical situation that the ground state $\Omega$ is the only vector (in the representation in question) invariant under time displacements. In a Lorentz invariant theory this is connected by the Lorentz group to the fact that $\Omega$ is the only vector invariant under space-translation; but here the two properties are independent. Thus the second was proved in the last section, using the cluster properties of the Wightman functions, but we have not succeeded in finding a proof of the first. We shall prove a weaker form in the next lemma, which is all that is needed for the Goldstone theorem.

Lemma 2. If $H$ is invariant under space translations, then there is no vector invariant under $U(t)$ of the form

$$
\Omega^{\prime}=\sum C_{l} J_{l}^{-} \Omega, \quad \sum\left|C_{l}\right|^{2}<\infty .
$$

Proof. If $H \Omega^{\prime}=0$ then $\sum C_{l}\left[H, J_{l}^{-}\right] \Omega=0$, giving from (18),

$$
\sum_{i, j}\left(C_{\imath} f_{i j}-C_{j} f_{i j}\right) J_{j}^{-} \Omega=0 \text {. }
$$

But all the $J_{j}^{-} \Omega$ are independent vectors, so that

$$
\sum_{i} C_{i} f_{i j}=C_{j} \sum_{i} f_{i j} \text { for all } j \text {. }
$$

Thus $C_{j}$ is the weighted mean of its neighbours, impossible if $f_{i j}$ all have the same sign and is translation invariant, unless all the $C_{j}$ are the same. But then $\sum\left|C_{l}\right|^{2}$ would diverge. This proves the lemma.

The next theorem shows that $\lim _{K \rightarrow \infty} J^{K}$ is, in some sense, the generator of a transformation which commutes with time-translations. In order to unify the proof in 1, 2 and 3 dimensions, we shall change the notation slightly. We assume we have a linear, square, or cubic array; a box containing the origin at the centre, and having size $(2 R)^{d}, d=1,2,3$ respectively, will be denoted by $V_{R}$. We assume that if $A \in \mathfrak{A}\left(V_{R}\right)$ then $[H, A] \in \mathfrak{P}\left(V_{R+q}\right)$, where $q$ measures the range of interaction. 
Theorem 7. Suppose $f(t)$ is a continuous function such that for any $N$ there is a number $F$ such that $|f(t)|<F(A+|t|)^{-N}$. Then if $A \in \mathcal{A}\left(V_{R_{0}}\right)$ we have

$$
\lim _{R \rightarrow \infty}\left(\Omega,\left[\underline{J}^{R}, A(f)\right] \Omega\right)=\left(\Omega,\left[\underline{J}^{R_{0}}, A\right] \Omega\right) \int f(t) d t
$$

where $A(f)=\int A(t) f(t) d t$ and $\underline{J}^{R}=\sum_{i \in V_{R}} \underline{J}_{i}$.

Proof. Since $A \Omega$ is analytic for the energy with infinite radius of convergence, we have

$$
\begin{aligned}
\left(\Omega,\left[\underline{J}^{R}, A(t)\right] \Omega\right)= & \left(\underline{J}^{R} \Omega, A(t) \Omega\right)-\left(A(t)^{*} \Omega, \underline{J}^{R} \Omega\right) \\
= & \lim _{n \rightarrow \infty}\left[\left(\underline{J}^{R} \Omega,\left\{A+i t H A+\cdots+\frac{i^{n} t^{n}}{n !} H^{n} A\right\} \Omega\right)-\right. \\
& -\left(\left\{A+i t H A+\cdots \frac{i^{n} t^{n}}{n !} H^{n} A\right\} * \Omega, \underline{J}^{R} \Omega\right) \\
= & \lim _{n \rightarrow \infty} \Omega,\left\{\left[\underline{J}^{R}, A\right]+i t\left[\left[\underline{J}^{R}, H\right], A\right]+\right. \\
& \left.\left.\left.+\cdots+\frac{i^{n} t^{n}}{n !}\left[\cdots\left[\underline{J}^{R}, H\right] \ldots H\right], A\right]\right\} \Omega\right) .
\end{aligned}
$$

Since $H_{H^{\prime}}$ is rotation invariant, $\underline{J}^{R}$ commutes with all the terms in (13), the expression for $H_{F}$, unless $i \in V_{R}, j \notin V_{R}$ or vice versa. But since the range of the forces is $q$, we see that $\left[H, \underline{J}^{R}\right] \in \mathfrak{A}\left(V_{R-q}\right)^{\prime}$. Then $\left[H,\left[H, J^{R}\right]\right] \in \mathscr{A}\left(V_{R-2 q}\right)^{\prime}$ etc. Here the dash denotes the commutant.

In fact, $\left[H \ldots\left[H, \underline{J}^{R}\right] \ldots\right]$ ( $k$ brackets) is in the algebra associated with the boundary of the box $V_{R}$, to a thickness $k q$ each side. Therefore $\left[H, \ldots\left[H, \underline{J}^{R}\right] \ldots\right]$ commutes with $A \in \mathfrak{A}\left(V_{R_{0}}\right)$ provided $R-k q>R_{0}$ i.e. $k<\left(R-R_{0}\right) / q$. Letting $n$ go to infinity in (21) the only non-zero terms, apart from $\left(\Omega,\left[\underline{J}^{R}, A\right] \Omega\right)$, are

$$
\begin{aligned}
\left(\Omega, \frac{i^{k} t^{k}}{k !}\left\{\left[\underline{J}^{R}, H^{k} A\right]\right.\right. & +\left[\underline{J}^{R}, H^{k+1} A\right] \frac{i t}{k+1}+ \\
& \left.\left.+\left[\underline{J}^{R}, H^{k+2} A\right] \frac{i^{2} t^{2}}{(k+1)(k+2)}+\cdots\right\} \Omega\right)
\end{aligned}
$$

with $k \geqq\left(R-R_{0}\right) / q$. For simplicity we choose $R$ so that $\left(R-R_{0}\right) / q$ is an integer, $k$.

Now $\left\|\underline{J}^{R}\right\|=\frac{1}{2}(2 R+1)^{d}$ for each component, since $\left\|J_{i}^{\alpha}\right\|=\frac{1}{2}$. Moreover $\left\|H^{k} A \Omega\right\| \leqq E^{k}\|A\|$, where $E$ is the maximum energy in $A \Omega$. Since $(k+n)^{-1}<n^{-1}$ we may bound (22) by

$$
2 \frac{\mid t^{k}}{k !} \frac{1}{2}(2 R+1)^{d} E^{k}\|A\| \exp (E|t|) .
$$

There exists an $F$ such that for all $t$

$$
|f(t)|<F(1+|t|)^{-6 q-2} .
$$

We may split $f$ into two parts, $f=f_{1}+f_{2}$, where

$$
\begin{aligned}
f_{1}(t) & =f(t), & & |t|<S=R^{1 / 2 q} \\
& =0, & & |t| \geqq S \quad \text { and } \\
f_{2}(t) & =0, & & |t|<S .
\end{aligned}
$$


We may bound $\int f_{2}(t) d t\left\{\left(\Omega,\left[\underline{J}^{R}, A(t)\right] \Omega\right)-\left(\Omega,\left[\underline{J}^{R}, A\right] \Omega\right)\right.$ easily by $2 \frac{1}{2}(2 R+1)^{d} 2 \int_{S}^{\infty}\|A\| F(1+|t|)^{-6 q-2} d t=\frac{2(2 R+1)^{l}}{6 q+1} F\left(1+R^{1 / 2 q}\right)^{-6 q-1}\|A\|$.

Since $d \leqq 3$ this goes to zero as $R \rightarrow \infty$. There remains

$$
\begin{aligned}
& \int_{-S}^{S} f_{1}(t) d t\left\{\left(\Omega,\left[\underline{J}^{R}, A(t)\right] \Omega\right)-\left(\Omega,\left[\underline{J}^{R}, A\right] \Omega\right)\right\} \leqq \\
& \quad \leqq \sup f(2 R+1)^{d} E^{k}\|A\| \frac{2}{k !} \int_{0}^{S} t^{k} \exp (E t) d t \leqq \\
& \leqq \sup f(2 R+1)^{d} E^{\left(R-R_{0}\right) / q} R^{\left(R-R_{0}\right) / 2 q^{2}} \exp \left(E R^{1 / 2 q}\right) /\left(\frac{R-R_{0}}{q}\right) ! \\
& \quad \rightarrow 0 \text { as } R \rightarrow \infty .
\end{aligned}
$$

The cases where $R \rightarrow$ but $\left(R-R_{0}\right) / q$ is not an integer can be proved along similar lines. This proves the theorem.

It follows immediately that if $f=\dot{g}$ or $\ddot{g}$ then

$$
\lim _{R \rightarrow \infty}\left(\Omega,\left[\underline{J}^{R}, A(f)\right] \Omega\right)=0 \text { for any local } A \text {. }
$$

Suppose now, if possible, that there is an energy gap in the states in $\mathscr{H}_{1}$, the states of one spin wave. We will show that this leads to a contradiction. Obviously lemma 2 implies that $\Omega$ is the only vector in $\mathscr{H}$ not orthogonal to all $\underline{J}^{R} \Omega$. We may therefore follow the proof of [15], lemma (iii), to prove the following

Lemma 3. For every local $A$ there exists a local $B$ and a continuous rapidly decreasing function $g(t)$ such that for all $R$

$$
\left(\underline{J}^{R} \Omega, A \Omega\right)=\left(\underline{J}^{R} \Omega, B(\ddot{g}) \Omega\right)+\left(\underline{J}^{R} \Omega, \Omega\right)(\Omega, A \Omega)
$$

if there is an energy gap in $\mathscr{H}_{1}$. Similarly

$$
\left(A^{*} \Omega, \underline{J}^{R} \Omega\right)=\left(B(\ddot{g})^{*} \Omega, \underline{J}^{R} \Omega\right)+\left(A^{*} \Omega, \Omega\right)\left(\Omega, \underline{J}^{R} \Omega\right) .
$$

This lemma immediately gives a contradiction to the hypothesis of a mass-gap. For using it

$$
\begin{aligned}
\left(\Omega,\left[\underline{J}^{R}, A\right] \Omega\right)= & \left(\underline{J}^{R} \Omega, B(\ddot{g}) \Omega\right)+\left(\underline{J}^{R} \Omega, \Omega\right)(\Omega, A \Omega) \\
& -\left(B(\ddot{g})^{*} \Omega, \underline{J}^{R} \Omega\right)-\left(A^{*} \Omega, \Omega\right)\left(\Omega, \underline{J}^{R} \Omega\right) \\
= & \left(\Omega,\left[\underline{J}^{R}, B(\ddot{g})\right] \Omega\right) .
\end{aligned}
$$

Hence

$$
\lim _{R \rightarrow \infty}\left(\Omega,\left[\underline{J}^{R}, A\right] \Omega\right)=\lim _{R \rightarrow \infty}\left(\Omega,\left[\underline{J}^{R}, B(\ddot{g})\right] \Omega\right)=0 .
$$

But by inspection the left-hand side is non-zero. This contradiction shows that the energy spectrum of states in $\mathscr{H}_{1}$ goes to zero, which is the statement of Goldstone's theorem. 


\section{On Certain Representations of $\mathfrak{a}$}

It is clear that the representation $\pi_{0}$ of $\mathfrak{A}$ corresponds to the zero temperature states, $\Omega$ being at absolute zero, and the other vectors in $\mathscr{H}$ being microscopic deviations from it. The inequivalent representations $\pi_{h}, h \in S U_{2}$, correspond to similar situations, but where there is a macroscopic difference in the lattice, namely all spins are pointing in a specific direction in the ground state, obtained from the third direction by rotating with $h$. Of the other inequivalent representations obtained from weak equivalence classes in the tensor product, most are too complicated to correspond to experimentally realizable situations. But those obtained from $\pi_{0}$ by inserting a finite amount of energy and "performing a large translation" [4] correspond to the various superselection sectors. Thus, for example, if $\psi_{i} \in \mathscr{H}_{i}$, and $\psi_{i}=|+\rangle_{i}$ in one half of the space, $\psi_{i}=|-\rangle_{i}$ in the other, then the magnet is divided into two magnetic domains by a Bloch wall. Let us denote the corresponding representation by $\pi_{1}$. The scattering of spin-waves from the domain boundaries, and the movement of the boundaries themselves, is then predicted by the vacuum sector dynamics, just as in [4] the behaviour of particles carrying superselection quantum numbers is derived from the dynamics of the observables alone, which are the primary objects of the theory. This

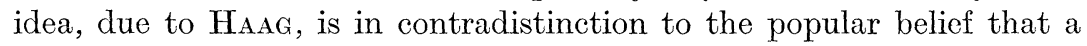
fundamental field theory of matter must involve the introduction of non-observable fields at a basic level.

One can easily see that the vacuum representation $\pi_{0}$ is recovered from $\pi_{1}$ by taking large translations [4].

Since in this model the spin-wave number $N$ is conserved in time, the thermodynamic states of the system should be given by

$$
\varrho_{\beta, \mu}(A)=\lim _{V \rightarrow \infty} \frac{\operatorname{tr}\left(e-\beta\left(H_{V}-\mu N\right) A\right)}{\operatorname{tr}\left(e-\beta\left(H_{V}-\mu N\right)\right)}
$$

where $H_{V}$ is some cut-off Hamiltonian such that the traces exist. The existence of this limit has been studied by D. W. RobInson, who has obtained results for a wide class of lattice systems, including those considered in this paper ${ }^{2}$. For $\beta=0\left(T=\frac{1}{\beta}\right.$ is the temperature $), \varrho(A)$ is given by the unique central state on $\mathfrak{A}$, producing a type $\mathrm{II}_{1}$ factor representation. The type III states discussed by R. T. Powers [23], defined by

$$
\left\langle J_{i}^{3}\right\rangle=\lambda, \quad\left\langle J_{i}^{1}\right\rangle=\left\langle J_{i}^{2}\right\rangle=0
$$

with no correlations between different spins, can be obtained by letting $\beta \rightarrow 0, \mu \rightarrow \infty$ such that $\mu \beta \rightarrow 2 \tanh ^{-1}(2 \lambda)$; but these states are more naturally obtained as the equilibrium states of a free spin system in an external magnetic field $\mu$. That is, we write a formal total Hamiltonian

${ }^{2}$ D. W. Robinson (private communication). 
$H_{F}=\mu \Sigma_{i} J_{i}^{3}$, and renormalize it by using (14). We obtain $H=\mu N$, and obtain (27) for the state at temperature $1 / \beta$, with $\mu \beta=\tanh ^{-1}(2 \lambda)$. This is in agreement with the ideas of Hugenholtz [24] who shows from general axioms (not all easy to check for the spin system) that the thermodynamic states give factors of type III except possibly for type $\mathrm{II}_{1}$.

If the limit (27) could be defined, giving rise to a translation invariant state, then we would say that the magnet is ferromagnetic at a temperature $\frac{1}{\beta}$ if $\lim _{\mu \rightarrow 0} \varrho\left(\beta, \mu, J_{i}^{3}\right) \neq 0$, and paramagnetic if $\lim _{\mu \rightarrow 0} \varrho\left(\beta, \mu, J^{3}\right)=0$.

As $\beta \rightarrow \infty$ in (27) the factor $e^{-\beta H}$ is very small for all states with any energy, so that in the trace only the vacuum survives, giving

$$
\varrho(\beta=\infty, A)=(\Omega, A \Omega) .
$$

This heuristically justifies the name "zero temperature state" for $\Omega$.

Acknowledgements. The author is indebted to T. W. B. KibBLE, J. P. MARChand, and A. Wuhlfson for stimulating conversations, and to L. Motchane for his kind hospitality at the I. H. E. S. where this work was completed.

\section{Literature}

1. HAAG, R.: In: Colloques sur les Problèmes Mathématiques de la Théorie Quantique des Champs. Paris: Centre des Recherches Scientifiques Nationales 1959.

2. -, and B. Schroer: J. Math. Phys. 3, 248 (1962).

3. Araki, H.: Local quantum theory, New York: Benjamin 1967.

4. Borchers, H. J.: Commun. Math. Phys. 1, 281 (1965).

5. See Feynman, R. P.: Lectures in physics. New York: Mc Graw Hill 1964.

6. Doplicher, S., D. Kastler, and D. W. Robinson: Commun. Math. Phys. $\mathbf{3 , 1}$ (1966).

7. Kastler, D., and D. W. Robinson: Commun. Math. Phys. 3, 151 (1966).

8. Ruelle, D.: Commun. Math. Phys. 3, 133 (1966).

9. TAKeda, Z.: Tohuku Math. J. 6, 212 (1954).

10. Segal, I. E.: Ann. Math. 63, 160 (1956).

11. Shale, D., and W. F. Stinespring: Ann. Math. 80, 365 (1964).

12. Guichardet, A.: Ann. Sci. Ecole Norm. Super. 83, 1 (1966).

13. Garding, L., and A. S. Wightman: Proc. Nat. Acad. Sci. 40, 617 (1954).

14. Goldstone, J., A. Salam, and S. Weinberg: Phys. Rev. 127, 965 (1962).

15. Kastler, D., D. W. Robinson, and J. A. Swieca: Commun. Math. Phys. 2, $108(1966)$.

16. Streater, R. F.: Proc. Roy. Soc. 287 A, 510 (1965).

17. von Neumann, J.: Comp. Math. 6, 1 (1938). Collected works, Vol. III, No. 6. New York: Pergamon 1961.

18. Klauder, J., J. McKenna, and E. J. Woods: J. Math. Phys. 7, 822 (1966).

19. Streit, L.: Commun. Math. Phys. 4, 22 (1967).

20. Streater, R. F., and A. S. Wightman: PCT, spin and statistics, and all that. New York: W. A. Benjamin 1964.

21. Nelson, E.: Ann. Math. 70, 572 (1959).

22. Streater, R. F.: In: Mathematical theory of elementary particles. Edited by I. E. Segal, and R. Goodman. Cambridge, Mass.; MIT Press 1966.

23. Powers, R. T.: Conference on $C^{*}$-algebras, Baton Rouge, 1967.

24. Hugenholtz, N. M.: Conference on $C^{*}$-algebras, Baton Rouge, 1967. 